\title{
Energy-Efficiency Improvements for Optical Access
}

\author{
Peter Vetter, Dusan Suvakovic, Hungkei Chow, Prasanth Anthapadmanabhan, \\ Konstantinos Kanonakis, Ka-Lun Lee, Fabienne Saliou, Xin Yin, and Bart Lannoo
}

\begin{abstract}
This paper discusses novel approaches to improve energy efficiency of different optical access technologies, including time division multiplexing passive optical network (TDM-PON), time and wavelength division multiplexing PON (TWDM-PON), point-to-point (PTP) access network, wavelength division multiplexing PON (WDM-PON), and orthogonal frequency division multiple access PON (OFDMA-PON). These approaches include cyclic sleep mode, energy-efficient bit interleaving protocol, power reduction at component level, or frequency band selection. Depending on the target optical access technology, one or a combination of different approaches can be applied.
\end{abstract}

Index Terms-Broadband communication, Optical fiber subscriber loops, Energy efficiency

\section{INTRODUCTION}

$\mathrm{E}$ NERGY consumption has become a major performance metric of communication systems in recent years. In response, several "green-networking" initiatives have been created. Typically they are calling for a near-term energyefficiency improvement target of tens of percent.(e.g., EU Code of Conduct [1]). GreenTouch, an international research consortium of operators, vendors, and academia, started in 2010 and aims at a more aggressive and longer-term target [2]. The present study was conducted in the context of the Wireline Access Working Group of GreenTouch. It is commonly agreed that the access network accounts for more than $80 \%$ of the total power consumption of a wireline network [1][3] and hence an important area of research on energy efficient networking. Fiber access systems are more efficient in terms of energy per transferred bit than other legacy technologies, such as their copper counterparts, because they feature a lower transmission loss and higher bandwidth. Previous papers in this magazine discussed how different fiber access

Manuscript received August 10, 2012.

P. Vetter, D. Suvakovic, H. Chow, P. Anthapadmanabhan are with Bell Labs, Alcatel-Lucent, Murray Hill, NJ 07974, USA. (e-Mail of corresponding author: peter.vetter@ alcatel-lucent.com).

K. Kanonakis is with AIT, Athens, Greece.

K.-L. Lee is with the Centre for Energy-Efficient Telecommunications (CEET), The University of Melbourne, Victoria, Australia.

F. Saliou is with Orange Labs, Lannion, France.

Xin Yin is with IMEC - Ghent University, Ghent, Belgium.

B. Lannoo is with Ghent University - IBBT, Ghent, Belgium.

This research was performed in the GreenTouch consortium. technologies exhibit different power consumption levels. Reference [4] reports an analysis of wavelength division multiplexing (WDM) passive optical network (PON) systems, while [5] provides a comprehensive survey of the power consumption by several candidate technologies for the nextgeneration passive optical network (NG-PON2) standard.

This paper examines several potential approaches, applicable to different fiber access technologies, for the purpose of lowering the power consumption at both near-term and long-term. Section II summarizes various optical access technologies and qualitatively discusses their energy efficiency. Section III gives an overview of the proposed power-saving approaches. While an approach may be suitable for a particular access technology, they can also be combined to obtain a better improvement. Section IV describes the model used to quantitatively compare different optical access technologies and analyzes the energy savings of the proposed approaches. Section V concludes the paper with a summary.

\section{OPTICAL ACCESS TECHNOLOGIES}

\section{A. TDM-PON}

In a traditional TDM-PON, an optical line termination (OLT) in the central office is connected to a number of optical network units (ONU) via a point-to-multipoint optical distribution network (ODN). In this architecture, the optical couplers are transparent and broad-spectrum power-splitters, usually simply referred to as "splitters." Gigabit-capable PON (ITU-T Recommendation series G.984) and Ethernet PON (IEEE Specification 802.3ah) are the most widely deployed fiber-to-the-home (FTTH) technologies today. They employ broadcast-and-select in the downstream and time division multiple access (TDMA) in the upstream direction. As an OLT interface is shared by multiple ONUs, TDM-PON represents the most energy-efficient optical-access solution currently deployed [3].

\section{B. Point-to-Point access}

Point-to-point (PTP) optical access systems currently deployed consume more power per subscriber than a TDMPON system, because every subscriber requires its own transceiver port at the OLT in the central office. However, in principle, PTP fiber should be the most energy-efficient in a wireline access network for the following two reasons: (1) subscribers are connected with the central office with a low 
attenuation fiber without any splitter losses; hence a lower optical power budget is sufficient; (2) while the ONUs of a TDM-PON are required to have high-speed optics and electronics that match the aggregated line rate of the PON, the line termination and user termination of the PTP connection can both operate at a lower speed that matches the subscriber's rate. The lower the operating speed, the lower the power consumption will be, because the dynamic power consumption of an electronic circuit is proportional to its operating frequency. The downside of the PTP approach, however, is the large fiber count in the distribution section. In deployments where the duct spaces are limited, a large fiber count becomes impractical.

\section{WDM-PON}

WDM-PON provides a virtual point-to-point fiber access connection via a dedicated wavelength to each ONU. All benefits mentioned in previous section on Point-to-Point access would apply to WDM-PON. However, in most deployments, a thermo-electric cooling (TEC) is often required to stabilize the wavelengths. A TEC consumes extra power, which diminishes any efficiency gain offered by its virtual PTP nature.

\section{TWDM-PON}

TDM-PON can be combined with wavelength division multiplexing (WDM) in a hybrid time wavelength division multiplexing PON (TWDM-PON). TWDM-PON increases the total capacity over the PON by the number of wavelength pairs, e.g., typically 4 x GPON or 4 x XG-PON1 (10 Gbit/s capable PON as specified in ITU-T G.987). Note that TWDMPON has been selected by Full Service Access Network (FSAN) as the primary solution for NG-PON2 (cf. ITU-T G.989). For the same total bandwidth capacity, the power consumption of an OLT will be slightly higher than a pure TDM-PON, because there are multiple transceivers at the OLT. On the ONU side, although the power consumption of the electronics is lower on a TWDM-PON, because the line rate per channel is lower as compared to the aggregated line rate for the TDM-PON, additional power is consumed on tuning the transmitter and receiver.

\section{E. OFDMA-PON}

The orthogonal frequency division multiple access PON (OFDMA-PON) recently gained interest in the research community as an alternative solution for next generation PONs [9][10]. OFDMA-PON employs a large number of subcarriers overlapping in spectrum, but not interfering with each other because they are orthogonal, hence achieving high spectral efficiency. OFDMA-PON offers a higher bandwidth granularity than WDM-PON.

As reported in [10], many flavors of OFDM transmission have been proposed for OFDMA-PON, including optical field modulation and intensity modulation (IM). At the receiver side, two detection methods have been proposed: coherent detection or direct detection (DD). Although field modulation coupled with coherent detection offers a better performance, it is considered as the most expensive, complex and powerhungry solution. For this reason, an IM/DD approach is chosen as a benchmark for the following analysis.

OFDMA-PON consumes additional power on digital signal processing (DSP) operations, e.g., Fast Fourier Transform (FFT) and Inverse FFT (IFFT). The requirement of high speed Analog to Digital and Digital to Analog Converters (ADCs/DACs) also contribute to its high power consumption. Note that in an IM/DD OFDMA-PON, the ADC and DAC have to operate at twice the expected sampling rate, since the use of IM implies that only half of the transmitted subcarriers can be used for carrying data [10].

\section{ENERGY EFFICIENCY IMPROVEMENTS FOR OPTICAL} ACCESS

\section{A. Sleep Mode}

Sleep mode is a method of power reduction by turning off parts of the system when the offered traffic is lighter than the total capacity of the system. The goal is to make the average power consumption as much as possible proportional to the traffic load. Most effort in research and standardization has been focused on implementing sleep mode in an ONU of a TDM-PON (e.g., [6]), but this technique can also be applied to the OLT on some access technologies.

For clarity, we define some of the terms used below. A state refers to a particular ONU condition where certain components, e.g., transmitter or receiver, are turned on or off. A mode generally consists of an ONU transitioning between at least two operating states.

In the active state, the ONU operates at full power with both the transmitter and receiver switched on, and thus data can be transmitted and received. A low-power state is a state in which parts of the system are turned off to reduce power consumption. For example, in the listen state, the transmitter is turned off while the receiver remains on, while in the sleep state both transmitter and receiver are turned off resulting in the lowest power consumption.

Various sleep mode mechanisms have been proposed for the ONU to enter and exit the low-power state(s). The transition can locally be triggered by the ONU when upstream traffic arrives at its user interface, but it is a more complicated problem for the ONU to know when to transition to the active state for the reception of downstream traffic from the OLT. Two approaches are commonly followed: (a) doze mode where the ONU transitions between the full-power active state and the low-power listen state; and (b) cyclic sleep mode where the ONU periodically transitions between the high-power active state and the low-power sleep state. The periodic transitions to an intermediate active state, which we call the probe state, allow the ONU for receiving an indicator from the OLT whether relevant downstream traffic is waiting at the OLT (Fig. 1). When the ONU receives an indicator that traffic is waiting, the ONU exits the sleep mode to the full-power active state. If there is no pending traffic, the ONU returns back to the low-power state and resumes the cyclic sleep mode. 
Different schemes are described in the literature to manage the state transition (e.g., [6][7]). In general, transitions to the "active state" are handled by a signaling protocol, while the periodic transitions within the cyclic sleep mode are handled autonomously by timers in the ONU.

As shown in Fig. 1, a sleep cycle consists of a relatively short probe state (typically less than a few milliseconds; we assumed $0.5 \mathrm{~ms}$ in our model below, i.e., four frames of $125 \mu \mathrm{s}$ ) followed by a longer period of sleep state (typically a few tens of milliseconds; we assumed $10 \mathrm{~ms}$ in our model). Increasing the length of the sleep state results in a trade-off between increased power saving and reduced quality of experience due to latency. We concluded from traffic simulations on XG-PON1 that durations of $5-10 \mathrm{~ms}$ in the sleep state and $0.5 \mathrm{~ms}$ in the probe state are a good compromise. There are no significant gains for longer periods in the sleep state. Another consideration is an increased memory size in the OLT and ONU for buffering respectively downstream and upstream traffic that arrives during the sleep state or listening state. Assuming a packet drop probability of less than $10^{-3}$, our traffic simulations showed that the memory size in the ONU to accommodate sleep period in the order of ten milliseconds is however limited. Our measurements on a prototype showed power savings of $30 \%$ using cyclic sleep mode. Estimates such as in ITU-T G Supplement 45 suggest that savings of more than $60 \%$ are possible. While most current solutions cyclically power switch of the transceiver, active development is underway by multiple suppliers to realize additional savings by dynamically managing the powerstate of other components and the power-state of functional blocks within large-scale system on chip (SoC). Moreover, research groups have been looking into protocol-compliant schemes (i.e., via proper medium access control (MAC) scheduling) to dynamically adjust individual ONU sleep state periods according to the actual traffic per ONU, in order to achieve a better trade-off between the energy-efficiency and quality of service (QoS) [6][7].

It is also possible to save energy in a PTP or WDM-PON optical access system by applying the cyclic sleep mode. Energy-Efficient Ethernet (EEE), which is specified in IEEE 802.3az for copper cables and backplanes, can conceptually also offer a mechanism for applying cyclic sleep mode to Ethernet PTP optical access networks. While in TDM-PON, cyclic sleep mode only applies to the ONU, ports at the OLT of a PTP system can also be powered down periodically. In its simplest implementation, transceivers will be shut off, but electronic circuitry will be kept alive in order to allow for a fast wake-up. A more sophisticated system may also power down parts of the electronic circuitry thereby trading off power consumption with delay time for waking up.

In addition to energy savings at the ONU, a quasi-static type of sleep mode can also be applied to the transceiver ports at the OLT of a TWDM-PON. The number of activated wavelength ports is matched the instantaneous traffic load, while other ports are turned off to save power. Such sleep mode scheme at the OLT occurs at a much longer time scale than at the ONU. It allows for achieving minimal power at the OLT when traffic is low for example during the night time, while fulfilling higher capacity demands during the day time.

It should be mentioned that hybrid OFDMA/TDMA operation is possible in OFDMA-PON, therefore sleep modes can also be exploited to increase energy efficiency. For the following analysis, we assumed that ADCs/DACs and up/down converters cannot be switched off during the sleep state.

\section{B. Bit Interleaving PON}

Bit Interleaving PON (BiPON) was developed in the context of GreenTouch as a new approach for energy-efficient TDM-PON. The idea behind the bit-interleaving protocol is that if an ONU can determine which bits are intended for other units, these bits should not undergo further processing, such as synchronization, de-scrambling, error-decoding, and deframing. In a TDM-PON, only a small fraction of the total bitrate is destined to a particalar ONU, therefore significant power savings can be realized.

As shown in Fig. 2, because the bits transmitted by an OLT are interleaved, only those intended for the ONU are sampled (also known as decimation from digital filtering). Hence immediately following the down-sampling, the original high speed at the input of the clock and data recovery (CDR) is reduced into a much slower user data rate, which is typically a few tens of Mbit/s for streaming services or services offered via a Wi-Fi interface (e.g., $54 \mathrm{Mbit} / \mathrm{s}$ for IEEE 802.11g), and occasionally $1 \mathrm{Gbit} / \mathrm{s}$ for fast downloads. Such user rate is much lower than the PON line rate typically at $10 \mathrm{Gbit} / \mathrm{s}$. As the information rate is significantly reduced, there is no need for a deserializer before further processing. The buffering requirements are minimized because there is no longer a need to store data bursts that arrives at a high speed on the PON interface. Slower clock speeds, smaller memory, and elimination of parallel processing requirements all contribute to an energy efficient design of an ONU. A BiPON ONU also exhibits lower static power consumption caused by transistor leakage than a conventional TDM-PON for two reasons: (1) a simplified processing results in a much smaller logic circuit design or less number of transistors; and (2) because the ONU design can be operated at slower clock, so it can be realized using a high-threshold CMOS transistor process. Slower clock speed also allows for more aggressive voltage scaling circuit design techniques, which further reduces the dynamic power dissipation.

To understand the bit-interleaving protocol, consider the frame structure as illustrated at the top of Fig. 2, where different colors denote bits being sent to different ONUs. The frame has a fixed length and consists of a header section and a payload section. Apart from the bit-interleaving organization, a major distinguishing feature of the BiPON protocol is its dynamic bandwidth allocation capability. Each ONU reads only its own bandwidth map encoded on its down-sampled header lane. The bandwidth map contains the down-sampling rate and offset information used for extracting the user bits in 
the payload section. Downstream bandwidth allocation can change every frame (every $125 \mu$ s in our design) matching the instantaneous traffic conditions.

We implemented the BiPON functionality for a $10 \mathrm{Gbit} / \mathrm{s}$ PON on an Altera Stratix IV field programmable gate array (FPGA). We chose this bitrate in order to compare it with the recently approved XG-PON1 standard, but the concept is evenly applicable to other rates. FPGAs are known to be energy-inefficient due to their relatively large static power consumption. To evaluate the energy efficiency of the design, we compare only the dynamic power consumption. We obtain the dynamic power consumption by subtracting the static (idle) power from its total power consumption. The dynamic power consumption for the electronic processing in a BiPON was measured at 10x lower than that of XG-PON1 implemented on the same FPGA platform. This results in a total reduction of $75 \%$ for the combination of transceiver and PON protocol functionality in the ONU.

\section{Efficiency improvements on optical transceiver}

It is possible to improve the energy efficiency of optical components in a PTP fiber access system by exploiting the low subscriber rate and optical budget. GreenTouch studies showed that the power consumption of a PTP transceiver at $1 \mathrm{Gbit} / \mathrm{s}$ can be reduced by more than an order of magnitude compared to a GPON transceiver (with $2.5 \mathrm{Gbit} / \mathrm{s}$ receiver and $1 \mathrm{Gbit} / \mathrm{s}$ transmitter) because of the reduced optical budget requirements. A PTP transceiver with an optimized transmitter and avalanche photodetector at $155 \mathrm{Mbit} / \mathrm{s}$ can even consume 70x less than a typical transceiver used in a GPON [8]. While operating at slower clock rates and lower laser currents, the PTP transceiver may use a simple low-voltage single-ended CMOS I/O driver, instead of the conventional current mode logic (CML) or low voltage differential signal (LVDS) which typically consume more power. By introducing programmable transmitter and receiver circuits, it is also possible to adaptively control the optical launch power and the corresponding receiver signal gain at the transimpedance amplifier (TIA) in both the OLT and ONUs to optimize their operating point.

These power saving approaches can also be applied to WDM PON systems, in which the virtual point-to-point link is transmitted over a wavelength channel instead of a dedicated fiber connection. An additional improvement on the component level is to employ a cooler-less tunable WDM laser or cooler-less reflective semiconductor optical amplifier (RSOA), which have the benefit of high-wavelength precision without using a power-hungry TEC.

\section{Energy efficient aggregation switch}

It is possible to improve the energy efficiency of the switching fabric in access systems by considering the relatively low network utilization and typical aggregation function. Specifically in today's PTP systems, the OLT is based on switched Ethernet technology, which was originally designed for many-to-many network connectivity with $100 \%$ duty cycle.
If the destination addresses are set hierarchically, a tree-based binary switch can efficiently and effectively forward downstream packets to the corresponding ONU (Fig. 3) without a complicated address lookup operation. A binary switch also enables upstream traffic aggregation without consuming energy on address lookup operation. We designed and simulated at gate level such bi-directional tree-based binary switch. Our model includes all necessary queues for mitigating upstream congestion. Our simulation results show that an optical access network with such binary switch could in theory consume less than $100 \mu \mathrm{W} /$ user (assuming a sustained traffic of $100 \mathrm{Mbit} / \mathrm{s}$ per subscriber). In other words, the power consumption of the switch becomes negligible compared to the total power consumption of all physical interfaces and interconnections.

\section{E. Band filtering}

In OFDMA-PON, it is possible to reduce the power consumption of an ONU by selecting a relevant set of contiguous subcarriers with a band pass filter. The ONU does not need to process the entire spectrum and can hence operate power hungry functions like ADC/DAC and DSP at much lower rates than the aggregate. For the BiPON in Section B, we aimed at selecting relevant bits early in the data flow in the time domain. Here, the same principle is applied in the frequency domain.

Fig. 4 depicts a simplified view of the main blocks contributing to the power consumption in an IM/DD OFDMAPON system, at the (a) receiving and (b) transmitting side of both OLT and ONU. For the performance evaluation, it was assumed that there are in total 64 data-carrying subcarriers in each direction. Each subcarrier employs differential quadrature phase shift keying modulation (DQPSK), with the bitrate per subcarrier being $156.25 \mathrm{Mbit} / \mathrm{s}$ (excluding a cyclic prefix (CP) equal to $12.5 \%$ of each OFDM symbol duration), thus resulting in an aggregate symmetrical bitrate of $10 \mathrm{Gbit} / \mathrm{s}$. Each ONU is able to use at maximum 16 subcarriers, which is the smallest contiguous set of carriers that we can realistically filter in an IM/DD OFDMA system.

It is important to note again that the ONU electronics (unlike the optics though) do not need to operate at the aggregate speeds. Thus, as shown in the figure, the ADC/DAC and FFT/IFFT blocks for the ONU are four times smaller than the respective ones for the OLT. This however implies extra power for down-converter/up-converter blocks. The rest of the electronics at the OLT/ONUs in the flow consume power comparable to XG-PON1/GPON digital circuitry respectively, due to their equivalent capacities.

\section{COMPARISON}

\section{A. Energy Consumption Model}

To compare the power consumption of different optical access technologies under different energy efficiency improvement techniques, we modeled the power consumption of all key building blocks inside the OLT and ONU. The 
results are broken down into downstream and upstream operation; each is further subdivided into digital processing and physical (PHY) layer.

Our power consumption model of an OLT includes digital processing and memory required for clock and data recovery (CDR), forward error correction (FEC), MAC and transmission convergence (TC) layer for shared medium PON, or an equivalent layer 2 (L2) aggregation of $64 \mathrm{ONU}$ for PTP and WDM-PON. Other OLT functions such as packet processing, traffic management, and L2 aggregation to the metro network interfaces are not considered, because they are common for all technologies. To estimate the power consumption per subscriber of the OLT on a typical deployment, we assumed $50 \%$ of the homes passed are connected on a PON using a splitting factor of 64. In other words, only 32 ONUs are served by an OLT on a PON.

The power consumption model of an ONU includes digital processing and memory required for CDR, FEC, MAC, and transmission convergence (TC) and L2 processing up to a Gigabit media independent interface (GMII) interfacing with other functions in the customer premises equipment (CPE). Again, the model does not include functions such as packet processing, traffic management, wired/wireless home network interfaces, voice interfaces, and routing gateway processing. Although some of these functions may be integrated on the same custom chip, they are not included in our model because they are common for all optical access technologies.

The transmitter model at the PHY includes the laser, laser driver, and other functions required for transmit operation (e.g., wavelength tuning, DAC, and modulation). The receiver model includes the photo detector, TIA, limiting amplifier, and other components required for receive operation (e.g., optical or electrical filter tuning, ADC, demodulation). For cases where a TEC is required, we assumed that the energy required to take away the heat load scales with the energy dissipated by the component (instantaneous power variations, e.g., due to cyclic sleep mode are averaged out by the slower thermal effects, but the proportionality holds when integrated over time). Though FFT and IFFT for OFDMA-PON are considered part of the PHY layer, their power consumption is modeled as part of the digital blocks, because they are implemented in CMOS technology.

The power consumption for each functional block is estimated using its recent datasheet published by the component vendors between 2010 and 2012. Values on the datasheet are specified for direct current (DC) voltage supply. We factored in an efficiency of $80 \%$ for the power supply. All custom chips are assumed to be manufactured in $28 \mathrm{~nm}$ CMOS technology. For those components/systems without data at our target operating speed, we scaled their power consumption with the projected performance. It is because a higher throughput in a custom chip typically would require a wider bus accordingly. In the case of Bi-PON, we measured the dynamic power consumption of our FPGA implementation and used it as an estimator for a full-custom ASIC implementation.
Similarly for OFDMA-PON, we estimated the dynamic power consumption by simulating the (I)FFT functions dimensioned with the system parameters described in Section III.E using Xilinx FPGA design tools. We used 64 data-carrying subcarriers in the ONU without band filtering and 16 subcarriers with band filtering. We assumed the ADC and DAC are $28 \mathrm{~nm}$ CMOS designs and estimated the power consumption to be $36 \mathrm{~mW} / \mathrm{GSps}$ and $16 \mathrm{~mW} / \mathrm{GSps}$, respectively. For ADC/DAC driver amplifiers operating at $10 \mathrm{GHz}$, we assumed $100 \mathrm{~mW}$ for their power consumption. Finally, for the up/down converters our best-guess estimate of $300 \mathrm{~mW}$ was chosen.

The following equations were used to model the power consumption of each component:

$$
\begin{aligned}
& P(r)=(1+a)(r / R) P_{\text {active }}+(1-(1+a) r / R) P_{\text {sleep }} \\
& P_{\text {sleep }}=b P_{\text {active }}
\end{aligned}
$$

$P(r)$ : Average power consumption as function of subscriber rate. The first term counts the portion of time when the component is in an active state, the second when the component is in a sleep state.

$P_{\text {active }}$ : Power consumption when component is transferring data at full capacity (active state),

$P_{\text {sleep }}$ : Power consumption when component is in a power saving sleep state,

$b$ : Proportional factor to estimate idle power as fraction of active power,

$r$ : Average subscriber rate (we modeled $50 \mathrm{Mbit} / \mathrm{s}$ downstream and $10 \mathrm{Mbit} / \mathrm{s}$ upstream, consistent with [1]),

$R$ : Maximum rate that a component is designed for (e.g., $2.5 \mathrm{Gbit} / \mathrm{s}$ downstream and $1.25 \mathrm{Gbit} / \mathrm{s}$ upstream in GPON, $1 \mathrm{Gbit} / \mathrm{s}$ for the user rate specific processing blocks),

a: Overhead (a ratio of listening state to total sleep cycle plus a preamble time for synchronizing the component).

Equation (1) is applied per component with its respective parameter values. Individual power consumption estimates are then summed together to obtain the total power consumption of a subsystem. Note that the actual power saving in the sleep state depends on the internal design of a component. Our model does not consider a probe state separately, but rather assumes that its power consumption is the same as the active state.

Table 1: Summary of parameters

\begin{tabular}{|l|l|}
\hline Parameter & Value \\
\hline$r:$ Subscriber rate & \\
$\quad-$ Downstream & $50 \mathrm{Mbit} / \mathrm{s}$ \\
$\quad-$ Upstream & $10 \mathrm{Mbit} / \mathrm{s}$ \\
\hline$R:$ Maximum rate of component & \\
$\quad-$ GPON & $2.5 \mathrm{Gbit} / \mathrm{s}$ \\
- PTP and WDM & $1 \mathrm{Gbit} / \mathrm{s}$ \\
- Other PON technologies & $10 \mathrm{Gbit} / \mathrm{s}$ \\
- User traffic specific block & $1 \mathrm{Gbit} / \mathrm{s}$ \\
\hline
\end{tabular}




\begin{tabular}{|c|l|}
\hline$b$ : Ratio idle power/total power & \\
- Digital electronics & 0.5 \\
- Transmitter & 0.2 \\
- Receiver & 0.0 \\
\hline$a:$ Overhead to synchronize component & \\
- Component for continuous bitstream & 0.1 \\
- Component for burst mode & 0.01 \\
\hline
\end{tabular}

Choices of parameter values (cf. Table 1$)$ :

$b=0.5$ as average for digital electronics. In [1] and from datasheets, values range from 0.3 to 0.6 between idle power and active power. Static power consumption in digital circuits is typically $30 \%$ for clock gated circuits. On the other hand, functional blocks like the internal clock, embedded processor and memory are assumed to be active all the time. We further more used $b=0.2$ for transmitter (to account for leakage in driver and power during silence time of a burst mode transmitter) and $b=0.0$ for receiver.

$a=0.1$ for continuous bitstream electronics and transceivers (PON downstream or Gigabit Ethernet point-to-point) - this is mainly to account for a $0.5 \mathrm{~ms}$ listening state in cyclic sleep mode with $10 \mathrm{~ms}$ period and an overhead time for electronics to synchronize.

$a=0.01$ for burst mode - this is $1 \%$ overhead counted for receiver synchronization or transmitter stabilization time for hardware that is designed for burst mode operation.

\section{B. Quantitative comparison}

Fig. 5 shows the comparison of the average power consumption per subscriber for different optical access technologies, with or without applying the respective power saving techniques described in Section III. We only discuss the PON variants with aggregate rates up to $10 \mathrm{Gbit} / \mathrm{s}$ rate and PTP systems of maximum $1 \mathrm{Gbit} / \mathrm{s}$, which is commonly sufficient for residential deployment. All cases support sustained rates of tens to a hundred Mbit/s and peak rates of at least one Gbit/s per subscriber. The average power consumption is computed over a busy hour period with a sustained rate of 50/10 Mbit/s using cyclic sleep mode. The comparison does not consider diurnal traffic cycles.

The power consumption of a XG-PON1 operating at $10 / 2.5 \mathrm{Gbit} / \mathrm{s}$ is higher than that of a GPON operating at 2.5/1.25 Gbit/s. For all cases, we can see that the sleep mode technique reduces the average power consumption primarily on the optical front end, but to a lesser extent on the digital electronics at the ONU. It is because part of the electronics has to remain active, e.g. the clocking circuit, processor, or memory to store data that arrived at a high line rate and is then forwarded to the user network interface at subscriber rate. There is no power saving at the OLT for these PONs for the sleep mode case, but its contribution is already small, because it is shared by many subscribers (32 in this calculation).

The bit interleaving protocol of $10 \mathrm{Gbit} / \mathrm{s}$ BiPON significantly reduces the power consumption of the electronics in an ONU, although the optical receiver is continuously on. The combination of BiPON with sleep mode will allow for turning off the receiver path intermittently for several frames. For example, 8 frames sleep cycle causes a delay of $1 \mathrm{~ms}$ while supporting $1.25 \mathrm{Gbit} / \mathrm{s}$ maximum throughput and lower user rates by decimation.

A standard Gigabit Ethernet PTP consumes more power per subscriber than GPON or XG-PON1, because there is an active port in the central office for each subscriber. The electronics at the ONU is less complex and hence consumes lower power. However, this is offset by the continuously on laser. A burst mode laser driver in a TDM-PON can be designed so that it consumes less power during silent period when it is not transmitting upstream data. Sleep mode can also bring a similar benefit to a PTP ONU and reduce the power consumption of a PTP OLT. The optimized approach for PTP as discussed in Section III.C and D results in the lowest power consumption.

Hybrid TWDM-PON multiplexing 4 x 2.5/1.25 Gbit/s achieves lower power consumption than XG-PON1, which features the same aggregate $10 \mathrm{Gbit} / \mathrm{s}$. More power is dissipated due to the optics in the OLT and heating to tune the transceiver in the ONU, but less in the electronics at the ONU. Sleep mode further reduces the power consumption of a TWDM ONU, while turning off three of the four wavelengths saves power at the OLT when traffic load is light.

The power consumption of WDM-PON is larger than a PTP system because of the WDM transmitters. At the OLT, the power consumption increases because of the TEC required for stabilizing the fixed wavelengths in a laser array. The ONU also consumes more power because a tunable laser is used, despite the fact that we assume no TEC and exploit the tunability for wavelength stabilization. The transmitter in WDM-PON consumes more than the one in TWDM-PON, because a tuneable laser with a wide tuning range is required for WDM-PON. In TWDM-PON, a simple distributed feedback (DFB) laser with heating is sufficient to cover the limited tuning range. As mentioned above, a power efficient design of the burst mode driver can save power during the silent periods. As with the PTP case, sleep mode reduces the average power consumption of the optical front ends in a WDM-PON. The power consumption of the TEC in the OLT scales with the average heat load that has to be carried away, even though it does not follow the same on-off sleep cycles. There are WDM-PON variants with a seeded wavelength and reflective semiconductor optical amplifier RSOA instead of a tunable laser in the ONU (e.g., [5]), for which similar sleep mode concepts can be applied.

OFDMA-PON has the highest power consumption per subscriber. The power consumption of its optical front end includes DAC and ADC and its digital electronics includes (I)FFT processing. The band filtering of the received signal in the downstream direction, as described in Section III.E, somewhat reduces the power consumption at the ONU, because the sampling rate of the ADC and the number of subcarriers to be processed by the FFT are reduced by a factor of four. Like other systems, sleep mode can offer additional 
benefits, but more modest compared to the other schemes.

\section{SUMMARY}

In this paper, we discussed several approaches to improve energy efficiency of different optical access technologies. Cyclic sleep mode is the most effective approach to reduce the power consumption when compatibility to existing standards is a given. In a TDM-PON, cyclic sleep mode applies to the ONU. The OLT is always on, but its contribution is relatively small, because it is shared by multiple subscribers. In an Ethernet PTP system, however, cyclic sleep mode can be applied to both the OLT port and ONU port.

When stepping away from the standards, Bi-PON combined with sleep mode is the most energy efficient system for a power splitting fiber plant. If the outside plant allows for a PTP fiber per subscriber, the most energy efficient access system is achieved by optimizing the aggregation switch and transceivers.

WDM-PON consumes more power due to the tunability of the WDM transmitter in the ONU, as well as the TEC for stabilizing the wavelengths at the OLT.

TWDM-PON offers an interesting capability to scale the power consumption of the OLT as a function of the required total capacity. It is done by lighting up an appropriate number of wavelength pairs accessible by all ONUs connected to the shared medium.

OFDMA-PON consumes the highest power due to the need for ADC/DAC, and (I)FFT. By narrowing the spectral band behind the ONU receiver, it is possible to reduce the power consumption incurred by the ADC and IFFT.

\section{ACKNOWLEDGMENT}

The authors would like to thank their colleagues and partners in the GreenTouch consortium for fruitful discussions.

\section{REFERENCES}

[1] European Commission, Joint Research Centre, "Code of Conduct on Energy Consumption of Broadband Equipment", Version 4.1, January 2013.

[2] GreenTouch, www.greentouch.org

[3] J. Baliga et al., "Energy consumption in wired and wireless access networks," IEEE Commun. Mag., pp.70-77, June 2011.

[4] K. Grobe, M. Roppelt, A. Autenrieth, J.-P. Elbers, and M. Eiselt, "Cost and Energy Consumption Analysis of Advanced WDM-PONs", IEEE Comm. Mag., pp. 25-32, February 2011.

[5] B. Skubic, E. In de Betou, T. Ayhan, and S. Dahlfort, "Energy-efficient next-generation optical access networks", IEEE Comm. Mag., pp. 122 127, January 2012.

[6] Jun-ichi Kani, "Power Saving Techniques and Mechanisms for Optical Access Networks Systems", Journal of Lightwave Technology, Vol. 31 Issue 4, pp.563-570, 2013.

[7] J. Zhang and N. Ansari, "Toward energy-efficient 1G-PON and 10GPON with sleep-aware MAC control and scheduling", IEEE Communications Magazine, pp. S33 - S38, February 2011.

[8] Ka-Lun Lee et al., "Energy efficiency of optical transceivers in fiber access networks", IEEE Journal of Optical Communications and Networking, Vol. 4 Issue 9, pp.A59-A68, 2012.

[9] N. Cvijetic, D. Qian and J. Hu, "100 Gb/s Optical Access Based on Optical Orthogonal Frequency-Division Multiplexing," IEEE Commun. Mag., pp. 70-77, July 2010.
[10] K. Kanonakis, et al., "An OFDMA-Based Optical Access Network Architecture Exhibiting Ultra-High Capacity and Wireline-Wireless Convergence", IEEE Commun. Mag, vol.50, no.8, pp.71-78, August 2012.

Peter Vetter (M'96) is Head of the Fixed Networks Research Program in Bell Labs. He received the degree of Physics Engineer from Gent University (Belgium) in 1986 and a $\mathrm{PhD}$ in 1991. After a post-doctoral fellowship at Tohoku University (Japan), he joined the research center of Alcatel (now Alcatel-Lucent) in Antwerp in 1993. He subsequently worked on liquid crystal displays, optical interconnections, optical access, access platforms, and access network architectures, first as researcher and later as department manager. In 2000, he became co-founder and R\&D lead for the PON technology in an internal venture that produced the first FTTH product in Alcatel. He also initiated and managed activities in various European Research Projects. From 2004-2008, he was the overall project manager of IST MUSE, a major European integrated project with 36 partners and covering all aspects of broadband access. Since 2009, he works at Bell Labs in Murray Hill, New Jersey, where he leads research on optical access, access platforms, and energy efficient access networks. He has authored or coauthored more than 90 international papers, including several invited. He has served in the technical program committees of ECOC, OFC, NOC, and BB Europe. He was the chair of the Wireline Access Working Group in GreenTouch for several years.

Dusan Suvakovic is a member of technical staff at the Bell Labs Access Solutions Research Department. His research interests include semiconductor issues in communication systems, low power implementation of network algorithms and protocols and optical access networks. He was one of the initial contributors to the Bell Labs green-ICT initiative. Dr. Suvakovic received his Ph.D. and M.A.Sc. degree in electrical and computer engineering from the University of Toronto, Canada and M.Sc. and B.Sc. degrees in electrical engineering at the universities of Belgrade and Novi Sad, Serbia. He has authored a number of technical papers and was granted 5 U.S. patents.

Hungkei (Keith) Chow is a member of technical staff in Access Solution Research Department at Alcatel-Lucent Bell Labs. He has contributed in diverse research areas including video and image processing, traffic and network management, copper and optical access network technologies. He has received the 2011 Bell Labs President's Award for his contribution in XTC: Crosstalk Cancellation and Phantom Mode Transmission. Dr. Chow received his $\mathrm{Ph} . \mathrm{D}$. in electrical and computer engineering from University of Toronto, Canada and a MPhil. degree from the Hong Kong University of Science and Technology in Hong Kong. He has authored many technical publications and has 5 patents granted, several pending.

N. Prasanth Anthapadmanabhan is a researcher at Alcatel-Lucent Bell Labs since 2010, where his research focuses on developing architectures and technologies for next generation wireline access networks. Previously, he was a Post-doctoral Fellow in the Wireless Networking and Communications Group at the University of Texas at Austin. He received the B.E. degree in electronics and instrumentation engineering from Birla Institute of Technology and Science, Pilani, India, and the M.S. and Ph.D. degrees both in electrical engineering from the University of Maryland, College Park. His research interests include information theory and applications, access networks, mobile backhaul and green communications.

Konstantinos Kanonakis was awarded his $\mathrm{PhD}$ degree in the field of optical networks from the National Technical University of Athens (NTUA), Greece, in 2008 and the Dipl.-Ing. Degree from the same university in 2004. His main research interests are in the area of design and analysis of architectures and control protocols for optical core and broadband access networks as well as their implementation in hardware. He has co-authored more than 50 papers that appeared in international peer-reviewed journals and conferences and has participated in several EU projects.

Dr. Kanonakis is a member of the IEEE, the IEEE Communications Society and the Technical Chamber of Greece.

Ka-Lun Lee received the Ph.D degree in electronic engineering from the Chinese University of Hong Kong, in 2003. His Ph.D. study included 
experimental study of short pulse generation, photonic analog to digital converter and multicasting of digital signal. In May 2004, he joined to the University of Melbourne (UoM) as a Research Fellow, and has been working actively in the areas of high speed multi-wavelength optical pulses generation for the application of ADC for optical label processing. In 2007-2010, he was with the Centre for Ultra-Broadband Information Networks (CUBIN), UoM and has been participating in an industry linkage project with NEC-Australia on extended-reach fiber access networks. Since April 2011, he is a senior research fellow with the Centre for Energy Efficient Telecommunications (CEET), UoM. He is currently developing new approaches for low energy fiber access networks. His research interest also includes optical wireless integrated access, microwave photonic, optical signal processing and electrooptic sampling.

Fabienne Saliou obtained a Ph.D degree in electronics and communications engineering from the University of Telecom Paris Tech in 2010. She joined Orange Labs - France Telecom R\&D in 2006 where she is currently a research engineer on next generation optical access networks. Her $\mathrm{Ph} . \mathrm{D}$ was focused on reach extension solutions for optical access networks and she contributed to many technical publications. She concentrated more recently her work on WDM PON technologies and the optimization of the energy efficiency of the optical access network within the internal program "Green ITN 2020". She is also collaborating in French, European and international projects such as JASMIN, ERMES, TREND and Greentouch consortium.

Xin Yin received the Ph.D. degree in electronic engineering from Ghent University, Belgium in 2009. Since 2007, he has been a researcher of IMECINTEC/Ghent University. His research interests include high-speed optoelectronic circuits and subsystems, with emphasis on burst-mode receiver and CDR for optical access networks, and low-power mixed-signal integrated circuit design for telecommunication applications.

Bart Lannoo received a MSc degree in electro-technical engineering and a PhD degree from Ghent University (Belgium) in July 2002 and May 2008, respectively. Since August 2002, he has been working at UGent-IBBT/INTEC in the IBCN (Internet Based Communication Networks and Services) research group, where he is currently a postdoctoral researcher. His current research interests are in the field of fixed and wireless access networks, focusing on MAC protocols, Green ICT and techno-economics. He has been involved in various national and European research projects. Since September 2011, Bart Lannoo is coordinating the Green ICT research at IBCN. He is author or coauthor of 70 national and international publications, both in journals and in proceedings of conferences. 


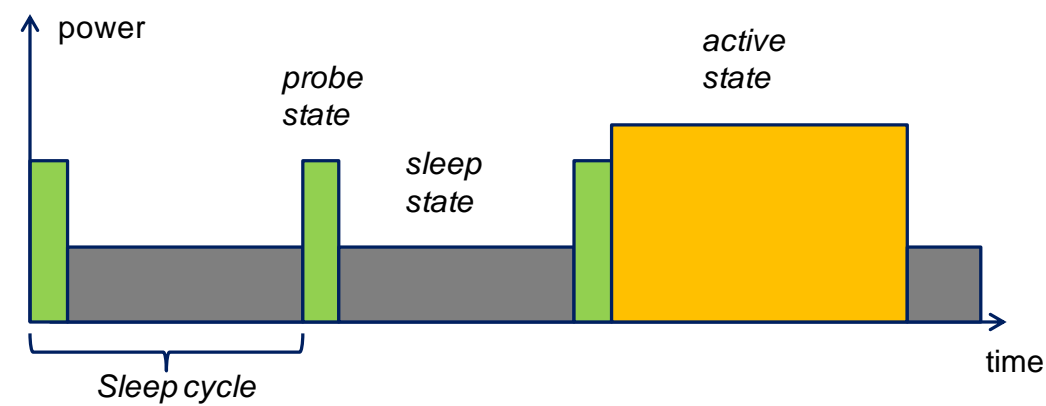

Figure 1. TDM-PON cyclic sleep mode. During extended periods when no customer traffic is present, a TDM-PON system can save energy by applying sleep states and cyclically probing for traffic in a listening state.

Frame structure (125 $\mu \mathrm{s})$

Interleaved bits broadcasted to all ONU

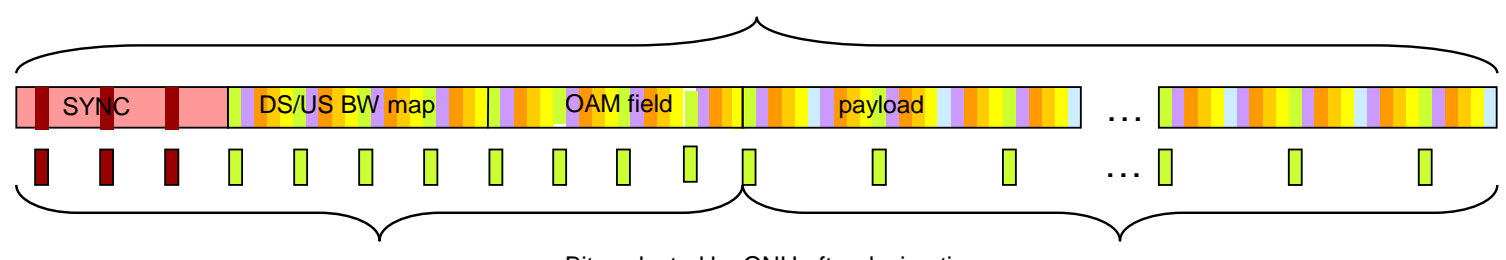

Bits selected by ONU after decimation

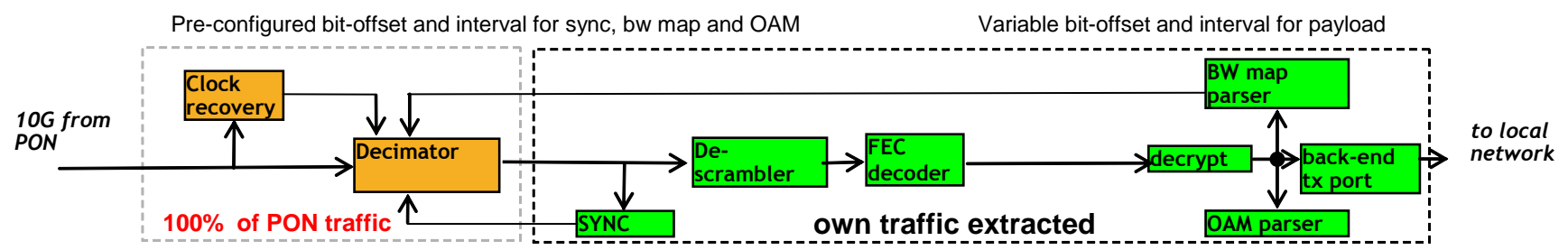

Figure 2. The frame structure of Bit Interleaved PON (top) allows for selection of relevant bits early in the data flow of an ONU (bottom) and minimizes the processing at full line rate.

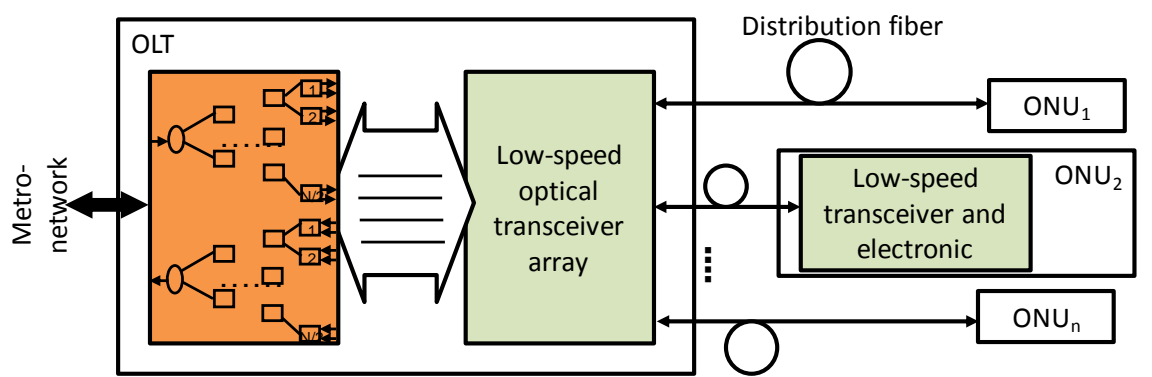

Figure 3. Minimum energy point-to-point optical access. 


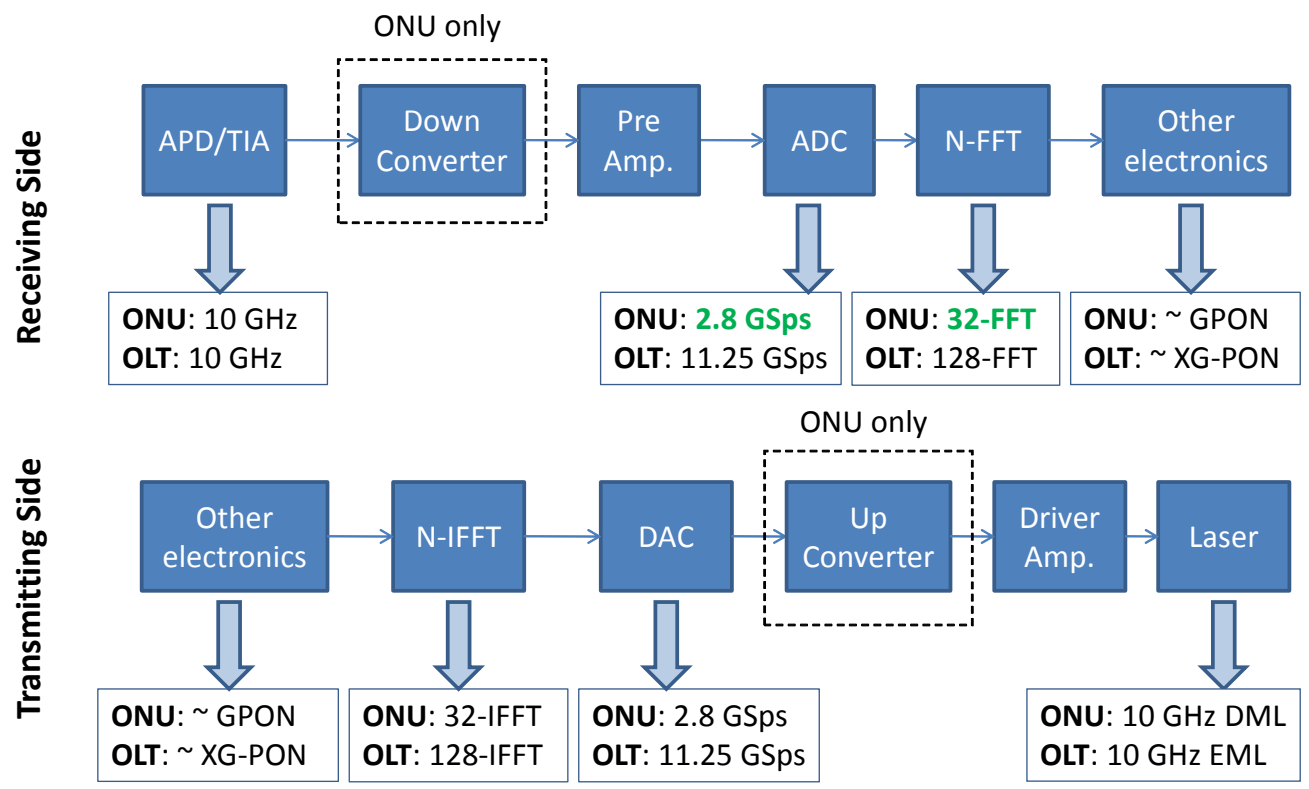

Figure 4. 10G IM/DD OFDMA-PON power consuming blocks in the band filtering case.

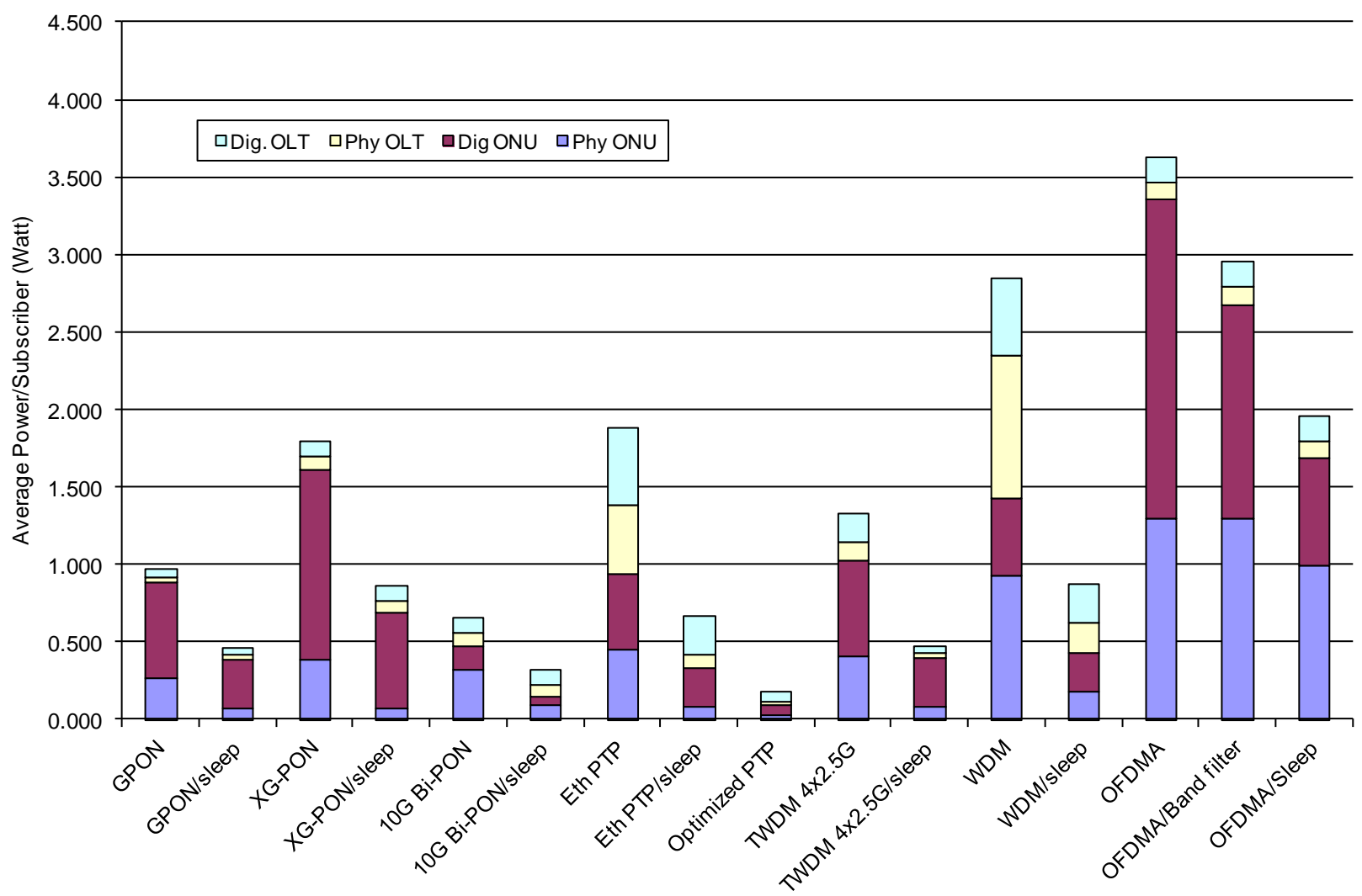

Figure 5. Average power consumption per subscriber for different technologies without and with their respective power saving approaches. 